\title{
MicroRNA-155-5p Targets NR3C2 to Promote Malignant Progression of Clear Cell Renal Cell Carcinoma
}

\author{
Chengquan Yan Pengfei Wang Chaofei Zhao Guangwei Yin Xin Meng \\ Lin Li Shengyong Cai Bin Meng \\ Department of Urology, Tangshan Gongren Hospital, Tangshan, PR China
}

\author{
Keywords \\ microRNA-155-5p - Nuclear receptor subfamily 3 group \\ C member 2 - Clear cell renal cell carcinoma - Malignant \\ progression
}

\begin{abstract}
Background: The molecular heterogeneity of clear cell renal cell carcinoma ( $c \mathrm{RCC}$ ) leads to a high mortality of the disease, which seriously threatens the life of patients. Therefore, this study explored the functional significance and mechanism of microRNA-155-5p and nuclear receptor subfamily 3 group $C$ member 2 (NR3C2) in the regulation of ccRCC. Methods: Expression levels of microRNA-155-5p and NR3C2 mRNA in ccRCC cells were analyzed by qRT-PCR, and the protein expression of NR3C2 in human cCRCC cells was measured by Western blot. Biological functions were determined through a series of in vitro experiments. The interaction between microRNA-155-5p and NR3C2 was tested by luciferase reporter gene assay. In addition, the effect of overexpressed or silenced microRNA-155-5p on cell phenotypes was evaluated in ccRCC cells. Results: Experimental data suggested that overexpression or silencing of microRNA$155-5 p$ in $c c R C C$ could boost or suppress cancer cell prolif-
\end{abstract}

Karger@karger.com www.karger.com/kbr

Karger $\stackrel{\text { ' }}{5}$

BOPEN ACCESS
(C) 2022 The Author(s)

Published by S. Karger AG, Basel

This is an Open Access article licensed under the Creative Commons Attribution-NonCommercial-4.0 International License (CC BY-NC) (http://www.karger.com/Services/OpenAccessLicense), applicable to the online version of the article only. Usage and distribution for commercial purposes requires written permission. eration and other malignant behaviors. Rescue experiments revealed that microRNA-155-5p facilitated the proliferation, migration, and invasion and suppressed the apoptosis of ccRCC by directly inhibiting the expression of NR3C2. Conclusions: This is the first study to generate new insights into the role of microRNA-155-5p/NR3C2 interaction in promoting the process of $\mathrm{CCRCC}$, and it is possible to bring a turning point for the treatment of ccRCC.

(C) 2022 The Author(s)

Published by S. Karger AG, Basel

\section{Introduction}

As the second most common type of cancer diagnosed in the human urinary system, renal cell carcinoma (RCC) accounts for about $3 \%$ of all malignancies and is the main cause of death with the incidence increasing year by year [1-3]. As a major subtype of RCC, clear cell RCC (ccRCC) occupies $70-80 \%$ of RCC [4] and imposes a serious impact on life quality of human beings. Currently, surgical resection is still the main treatment for ccRCC, but there are still nearly $40 \%$ of patients with recurrence or distant metastasis after treatment. Despite notable advances in surgical treatment recently, ccRCC patients' survival re-
Correspondence to:

Bin Meng, binmeng5511@163.com 
mains unsatisfactory $[5,6]$. Therefore, it is urgent to further understand the mechanism of ccRCC tumorigenesis and progression and to find more effective treatment methods for ccRCC patients.

As a class of small noncoding RNAs, microRNAs consist of 18 24 nucleotides and can negatively modulate their target genes through directly binding to the $3^{\prime}$ UTR of target genes [7], thus playing a critical regulatory part in physiological and pathological processes of the human body [8]. In recent years, with the deepening of research on the function of microRNA, it was confirmed that microRNA also plays a key part in the occurrence and progression of ccRCC. For example, Xie et al. [9] uncovered that microRNA-363 inhibits the proliferation, invasion, and migration of ccRCC cells via downregulating S1PR1. In addition, Liu et al. [10] also found that microRNA-935 facilitates the invasion and migration of ccRCC cells by targeting IREB2. However, several researchers unveiled that microRNA-155-5p on chromosome 21 regulates the progression of various cancers. For example, Chen et al. [11] confirmed that increased expression of microRNA155-5p can boost apoptosis of hepatocellular carcinoma cells by downregulating CThrC1 while inhibiting cell cycle progression, cell proliferation, cell invasion, and cell migration. Ning Li [12] found that microRNA-155-5p can induce the metastasis of cervical cancer, and its regulatory function is realized through the inhibition of TP53INP1. However, the molecular regulation mechanism of microRNA-155-5p in ccRCC remains unclear and needs to be further explored.

In the current study, we observed whether microRNA155-5p was differentially expressed in ccRCC cells and normal renal epithelial cells, as well as the effect of microRNA-155-5p on the phenotype of ccRCC cells through a series of functional experiments. Also, the downstream molecular regulatory mechanism of microRNA-155-5p in ccRCC was further explored. The results of this study will provide a theoretical basis for applying microRNA$155-5 \mathrm{p}$ as a potential molecular therapeutic target for patients with ccRCC.

\section{Materials and Methods}

\section{Bioinformatics Analysis}

The data of microRNA expression level (normal: 71, tumor: 545) and mRNA expression level (normal: 72, tumor: 539) of ccRCC were downloaded from TCGA database (https://portal. gdc.cancer.gov/) on November 29, 2019. A T test was applied to determine the expression of microRNA-155-5p in normal tissue and ccRCC tissue. Then, starBase (http://starbase.sysu.edu.cn/), TargetScan (http://www.targetscan.org/vert_72/), mirDIP (http://
ophid.utoronto.ca/mirDIP/index.jsp\#r), and miRDB (http:// mirdb.org/) were employed to predict the downstream regulatory target genes of microRNA-155-5p. In addition, the R package "edgeR" was used to analyze the difference in the mRNA expression data between the normal group and the tumor group $(\log |\mathrm{FC}|$ $>1.5, \mathrm{FDR}<0.05)$, and the results obtained were intersected with the differential mRNAs. Subsequently, the Pearson correlation analysis was applied to finally determine the target mRNA regulated by microRNA.

\section{Cell Culture and Transfection}

Human proximal renal tubular epithelial cell line HK2 (ATCC ${ }^{\circledR}$ CRL-2190) and human ccRCC cell lines 786-O (ATCC ${ }^{\circledR}$ CRL1932), A498 (ATCC ${ }^{\circledR}$ HTB-44), ACHN (ATCC ${ }^{\circledR}$ HTB-1611), and Caki-1 (ATCC ${ }^{\circledR}$ HTB-46) were purchased from ATCC. The HK2 cell line was cultured in Defined Keratinocyte SFM (Gibco, Billings, MT, USA). The 786-O cell line was cultured in RPMI-1640 medium. A498 and ACHN cell lines were cultured in DMEM. The Caki-1 cell line was cultured in McCoy's 5A medium. All media contained 10\% FBS (Gibco) and 1\% penicillin and streptomycin (Invitrogen, Waltham, MA, USA). All cells were cultured in a $37^{\circ} \mathrm{C}$ moist incubator with $5 \% \mathrm{CO}_{2}$.

MicroRNA-155-5p mimic, microRNA-155-5p inhibitor, shnuclear receptor subfamily 3 group C member 2 (NR3C2), and their corresponding negative controls (NC-mimic, NC-inhibitor, and sh-NC) were ordered from RiboBio (Guangzhou, China). The transfection was performed using Lipofectamine 2000 reagent (Invitrogen) according to the manufacturer's instructions.

\section{RNA Extraction and $q R T-P C R$}

Total RNA was isolated from 5 cell lines using TRIzol reagent (Invitrogen) for cDNA synthesis. Quantitative determination of mature microRNA and mRNA (microRNA-155-5p [accession No. AP000223] and NR3C2 [accession No. AB209056]) was performed using TaqMan microRNA (Applied Biosystems Inc., Foster City, CA, USA). U6 and GAPDH were applied as endogenous controls. qRT-PCR was performed using the QuantStudio 7 PCR system and Taqman universal PCR premix. The expression of microRNA-155-5p or NR3C2 was standardized. The primers used are shown in Table 1.

\section{Western Blot}

After $72 \mathrm{~h}$, cells were collected and lysed using NP-40 (Thermo Scientific). The Bicinchoninic Acid Protein Assay (Thermo Scientific) was used to measure the protein concentration. NuPAGE 4-12\% Bis-Tris protein gel (Invitrogen) was used for Western blot. For gel electrophoresis, the iBlot 2 Dry Blotting System (Invitrogen) was used to transfer proteins to a polyvinylidene fluoride membrane. Next, the membrane was incubated with Odyssey blocking buffer and then incubated overnight with primary antibodies at $4^{\circ} \mathrm{C}$. Then, the membrane was incubated with goat antirabbit IgG H\&L (HRP) (ab6721, 1:8,000; Abcam, Cambridge, UK) for $45 \mathrm{~min}$ at room temperature and then washed with TBST. The infrared imaging system scanner was used to generate Western blotting images. The primary antibodies used are as follows: NR3C2 (21854-1-AP, 1:1,000; Proteintech, Wuhan, China) and GAPDH (10494-1-AP1, 1:5,000; Proteintech).

\section{CCK-8}

The CCK8 cell proliferation assay kit (Dojindo Laboratories, Kumamoto, Japan) was employed to determine the proliferative 


\begin{tabular}{ll}
\hline Gene & Primer sequence \\
\hline microRNA-155-5p & $\begin{array}{l}\text { Forward: 5'-GATCAAAGTCTTCAAATATGCCTAAAGG-3' } \\
\text { Reverse: 5'-TGAACAAGCCAAAACCTGC-3' }\end{array}$ \\
\hline U6 & Forward: 5'-GCTTCGGCAGCACATATACTAA-3' \\
& Reverse: 5'-AACGCTTCACGAATTGCGT-3' \\
\hline NR3C2 & Forward: 5'-CACAGCACTGGTTCCTCAG-3' \\
& Reverse: 5'-TTGCCTGCTAAGCGGTTGA-3' \\
\hline GAPDH & Forward: 5'-TGTGTCCGTCGTGGATCTGA-3' \\
& Reverse: 5'-CCTGCTTCACCACCTTCTTGA-3' \\
\hline
\end{tabular}

capacity of the transfected cells. The $100 \mu \mathrm{L}$ suspension with transfected cells was inoculated into a 96-well plate. At specific time point of $0,24,48$, and $72 \mathrm{~h}, 10 \mu \mathrm{L}$ of CCK 8 reagent was added to each well (be careful to avoid bubbles). $2 \mathrm{~h}$ after reagent addition, the absorbance was measured at $450 \mathrm{~nm}$ with a microplate reader.

\section{Scratch Healing Assay}

Before inoculating cells, a marker pen was used to draw a horizontal line on the back of the 6-well plate (to facilitate the positioning of the same field of vision during photographing). Cells were transferred and then seeded into 6-well plates. When cells covered the bottom of the hole, monolayer cells were scraped with a sterile $10 \mu \mathrm{L}$ micropipette. The cell fragments generated by scratching were removed, and then, serum-free medium was added. The wound areas of the cells were observed and photographed at $0 \mathrm{~h}$ and $48 \mathrm{~h}$. Cell migration rate $=\left[1-\left(\right.\right.$ wound area at $\mathrm{T}_{\mathrm{t}} /$ wound area at $\left.\left.\mathrm{T}_{0}\right)\right] \times 100 \% . \mathrm{T}_{0}$ represented the time immediately after scratching, and $\mathrm{T}_{\mathrm{t}}$ represented the time $48 \mathrm{~h}$ after scratching.

\section{Transwell Invasion Assay}

Fifty microliter Matrigel (BD Biosciences, San Jose, CA, USA) was coated on the upper layer of the insert at $4^{\circ} \mathrm{C}$. Cells were incubated for $12 \mathrm{~h}$ without serum and were then washed and seeded into the upper chamber with serum-free bovine serum albumin (Invitrogen). $500 \mu \mathrm{L}$ DMEM high-glucose medium with 20\% FBS was placed in the lower chamber. Twenty-four hour later, the Matrigel and cells in the upper chamber were removed with a cotton swab. The remaining cells were fixed with $4 \%$ paraformaldehyde and stained with $0.1 \%$ crystal violet. The average cell number was counted in 5 randomly selected fields under an upright microscope.

\section{Cell Apoptosis Assay}

Cells were collected $72 \mathrm{~h}$ after transfection. The cells were washed with cold PBS, blown, and beat with a pipette to be fully resuspended in a binding buffer. Under dark conditions, the cells were stained with Annexin V-FITC and propidium iodide. Fifteen minutes later, the cells were washed and analyzed using BD FACSVerse (BD Pharmingen).

\section{Dual-Luciferase Assay}

To evaluate the mRNA that may be targeted by microRNA155-5p, the bioinformatics database was used to search for genes that can bind to microRNA-155-5p. Luciferase reporter vectors were constructed by ligation with a custom NR3C2 oligonucleotide containing the putative binding sites and the corresponding nontarget/mutation sites and were then inserted into pmiR-GLO reporting vector (Promega, Madison, WI, USA). HEK-293T cells treated with microRNA-155-5p mimic and negative controls were co-transfected with either NR3C2 wild type or NR3C2 mutant. Luciferase activity was detected using the Dual-Luciferase Reporter Gene at $48 \mathrm{~h}$ after transfection. The relative activity was calculated by normalized renilla luciferase.

\section{Statistical Analysis}

All assays were conducted with 3 biological replicates and were expressed to be mean \pm standard deviation. GraphPad Prism6 was applied for statistical analysis using Student's $t$ test. $p<0.05$ was considered significant.

\section{Results}

\section{MicroRNA-155-5p Is Highly Expressed in ccRCC}

It was reported that microRNA-155-5p expression is enhanced in various tumors and promotes malignant progression of cancer cells [12-14]. Therefore, based on the data of TCGA, we deduced that microRNA-155-5p expression was also upregulated in ccRCC tissue (Fig. 1a). Compared to normal renal epithelial cells (HK2), four ccRCC cell lines had significantly elevated microRNA155-5p expression, which further confirmed the upregulation of microRNA-155-5P in ccRCC (Fig. 1b). Through these experimental results, we fully proved that microRNA-155-5p was highly expressed in ccRCC.

\section{MicroRNA-155-5p Promotes Malignant Progression of ccRCC}

To further evaluate the regulatory function of microRNA-155-5p on ccRCC, we first transfected microRNA155-5p-inhibitor and NC-inhibitor (negative control) into 


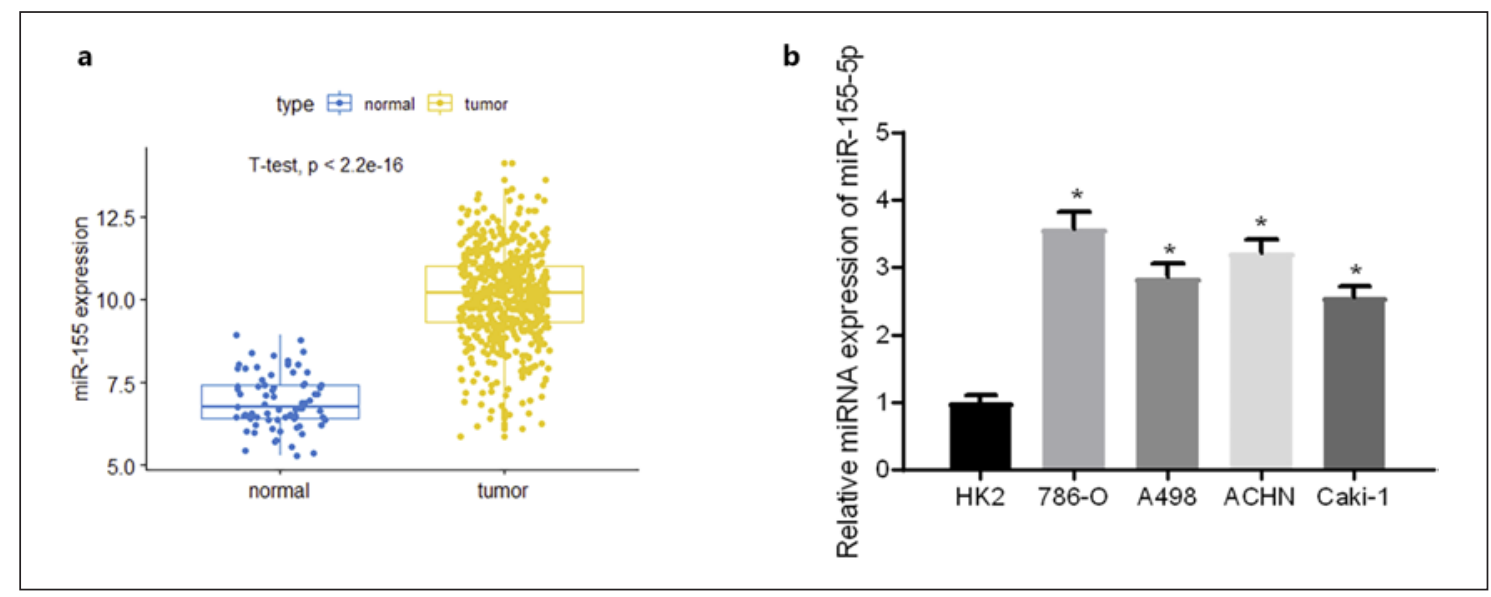

Fig. 1. MicroRNA-155-5p is highly expressed in ccRCC. a Expression level of microRNA-155-5p in cancer tissue and para-cancerous tissue of 42 patients with ccRCC detected by qRT-PCR. b Detection of microRNA-155-5p expression in HK2 and ccRCC cell lines. *denotes $p<0.05$.

786-O cells with relatively high expression of microRNA155-5p. Meanwhile, Caki-1 cells with relatively low microRNA-155-5p expression were treated with the microRNA-155-5p mimic for overexpressing microRNA-155-5p. In addition, Caki-1 cells were transfected with the NCmimic as the negative control. qRT-PCR confirmed that the transfection efficiency of each treatment group was in line with expectations, indicating that the transfected cells could be used for subsequent experiments (Fig. 2a). Then, the effects of microRNA-155-5p silencing and microRNA155-5p overexpression on the proliferation, migration, invasion, and apoptosis of ccRCC cells were detected by cell biological functional assays. CCK-8 results expressed that silenced microRNA-155-5p notably suppressed the proliferation of 786-O cells, while overexpressed microRNA$155-5 p$ prominently boosted the proliferation of Caki-1 cells (Fig. 2b). Meanwhile, scratch healing and Transwell assays suggested that silence of microRNA-155-5p reduced the migration and invasion of 786-O cells while overexpression of microRNA-155-5p enhanced the migration and invasion of Caki-1 cells (Fig. $2 \mathrm{c}-\mathrm{d}$ ). In addition, silenced microRNA-155-5p promoted the apoptosis of 786-O cells, while overexpressed microRNA-155-5p decreased the apoptosis of Caki-1 cells in the cell apoptosis assay (Fig. 2e). These results strongly indicated that microRNA-155-5p could induce malignant progression of ccRCC.

\section{NR3C2 Is a Direct Target of microRNA-155-5p}

In order to explore the downstream molecular mechanism of microRNA-155-5p, we next identified potential targets to clarify the functional role of microRNA-155-5p. Target predictions in silico was carried out by adopting 4 different algorithms - starBase, TargetScan, miRDB, and mirDIP. starBase provides information on the interaction between microRNAs and various RNA molecules, and it predicted 2,222 target genes for microRNA-155-5p. Customized TargetScan predicted 556 target genes for microRNA-155-5p. miRDB contains selected and possible microRNA targets, and it predicted 701 target genes for microRNA-155-5p. mirDIP contains the most comprehensive human microRNA target genes, and it predicted 1,447 target genes for microRNA-155-5p. To increase the rigor of the prediction, we limited potential targets to the four algorithms. Finally, a total of 215 target genes were obtained (Fig. 3A). Then, differential analysis was further performed on the data set of mRNA expression levels of ccRCC in TCGA, finding 2,468 mRNAs were upregulated and 1,165 mRNAs were downregulated in tumor tissue (Fig. 3B). Then, based on the negative regulatory mechanism of microRNA-mRNA in ceRNA, 1,165 downregulated mRNAs were intersected with 215 mRNAs predicted by the database, and finally 5 target mRNAs that had binding sites with microRNA-155-5p were gained (Fig. 3C). Correlation analysis revealed that NR3C2 had the highest negative correlation with microRNA-155-5p (Fig. 3D). Meanwhile, survival analysis of patients with ccRCC showed that low expression of NR3C2 was markedly detrimental to the prognosis of patients (Fig. 3E). Therefore, we finally chose NR3C2 as the study object. The specific expression of NR3C2 in tumor tissue in 
TCGA database is shown in Figure 3F. To confirm that $\mathrm{NR} 3 \mathrm{C} 2$ is the downstream target of microRNA-155-5p, the expression levels of HK2 and ccRCC cell lines were tested by qRT-PCR and Western blot. The results indicated that the NR3C2 expression level in ccRCC cells was prominently lower than that in normal renal epithelial cells (Fig. 3G). In addition, the targeting between microRNA-155-5p and NR3C2 was detected by the dual-luciferase assay. The results suggested that microRNA-155-5p prominently reduced the luciferase activity of the NR3C2WT reporter gene. This indicated that microRNA-155-5p could target NR3C2 (Fig. 3H). Finally, we also observed the expression of NR3C2 in 786-O cells with silenced microRNA-155-5p and Caki-1 cells with overexpressed microRNA-155-5p, finding that silenced microRNA-155-5p notably upregulated NR3C2 in ccRCC (Fig. 3I). However, overexpressed microRNA-155-5p remarkably downregulated NR3C2 in ccRCC (Fig. 3J). The above experimental results proved that NR3C2 was the target gene of microRNA-155-5p, and microRNA-155-5p could negatively regulate the expression of NR3C2.

\section{MicroRNA-155-5p Restrains ccRCC Malignant \\ Progression by Targeting NR3C2}

To determine whether microRNA-155-5p modulates the progression of ccRCC by targeting NR3C2, 786-O cells were transfected with the following vector groups: $\mathrm{NC}$-inhibitor + sh-NC (negative control), microRNA155-5p-inhibitor + sh-NC, and microRNA-155-5p-inhibitor + sh-NR3C2. The mRNA and protein expression levels of NR3C2 in 786-O cells of the 3 groups were detected by qRT-PCR and Western blot. The results suggested that sh-NR3C2 reversed the effects of microRNA155-5p-inhibitor on NR3C2 mRNA and protein in ccRCC cells (Fig. 4A). After that, the proliferation, migration, invasion, and apoptosis of 786-O cells in 3 groups were observed by cell biological functional experiments. It was found that the transfection of sh-NR3C2 markedly overturned the inhibitory effects of the microRNA-155-5pinhibitor on the proliferation of ccRCC cells (Fig. 4B).

Fig. 2. MicroRNA-155-5p promotes the malignant progression of ccRCC. a Expression level of microRNA-155-5p in 786-O and Caki- 1 cells detected by qRT-PCR. $\mathbf{b}$ Cell proliferative capacity of different treatment groups determined by the CCK- 8 assay. c Cell migratory ability in each treatment group detected by scratch healing assay $(\times 40)$. d Cell invasive ability of each treatment group detected by the Transwell assay $(\times 100)$. e Apoptosis of each treatment group detected by the cell apoptosis assay. ${ }^{*}$ denotes $p<0.05$. NC, negative control.
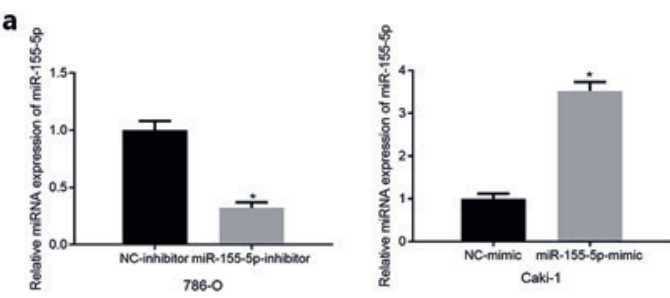

b
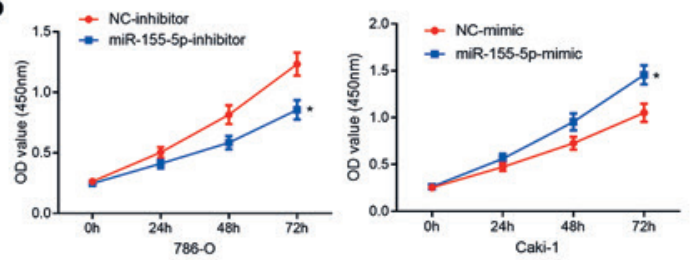

c
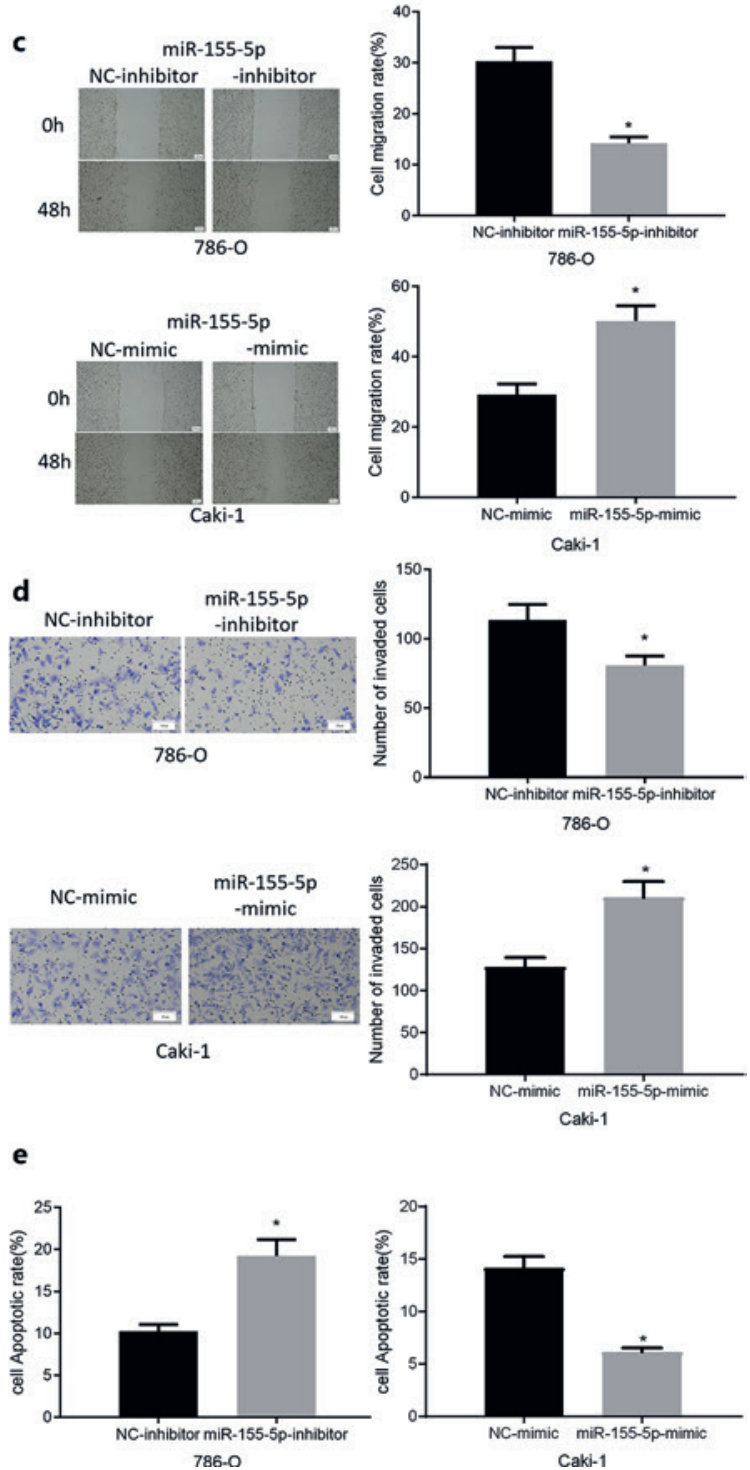
Fig. 3. NR3C2 is the target gene of microRNA-155-5p. A Venn diagram of target genes of microRNA-155-5p. B Volcano map of differential genes in the ccRCC mRNA data set in TCGA database between the normal group and the tumor group. Red and green dots represent upregulated and downregulated mRNA expression in ccRCC tissue, respectively. C Venn diagram of downregulated genes in ccRCC and predicted target genes of microRNA155-5p. D Pearson correlation heat map of microRNA-155-5p and its target genes. E Effect of NR3C2 expression on prognosis of ccRCC patients. $\mathbf{F}$ NR3C2 expression in TCGA database is downregulated in the tumor group. G Expression of NR3C2 mRNA and protein in HK2 and ccRCC cell lines. H Binding of microRNA-155-5p and NR3C2 detected by the dual-luciferase assay. I, J Effects of silencing or overexpressing microRNA-155-5p on NR3C2 expression in ccRCC cells. *denotes $p<0.05$. NC, negative control.

MicroRNA-155-5p Promotes Malignant Progression of ccRCC

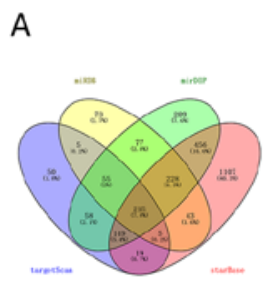

D

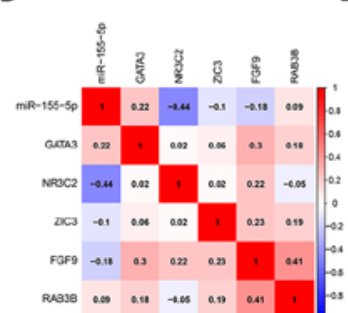

E

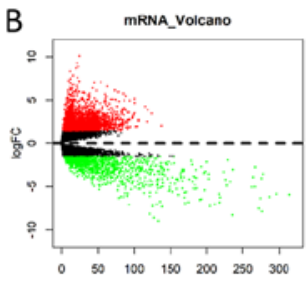

C
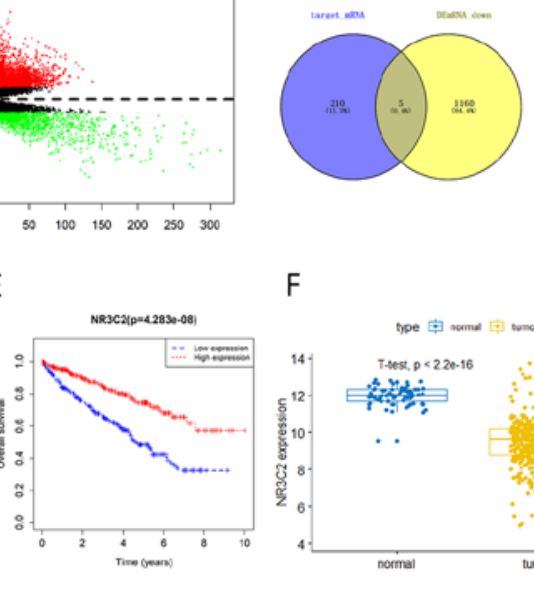

$\mathrm{F}$
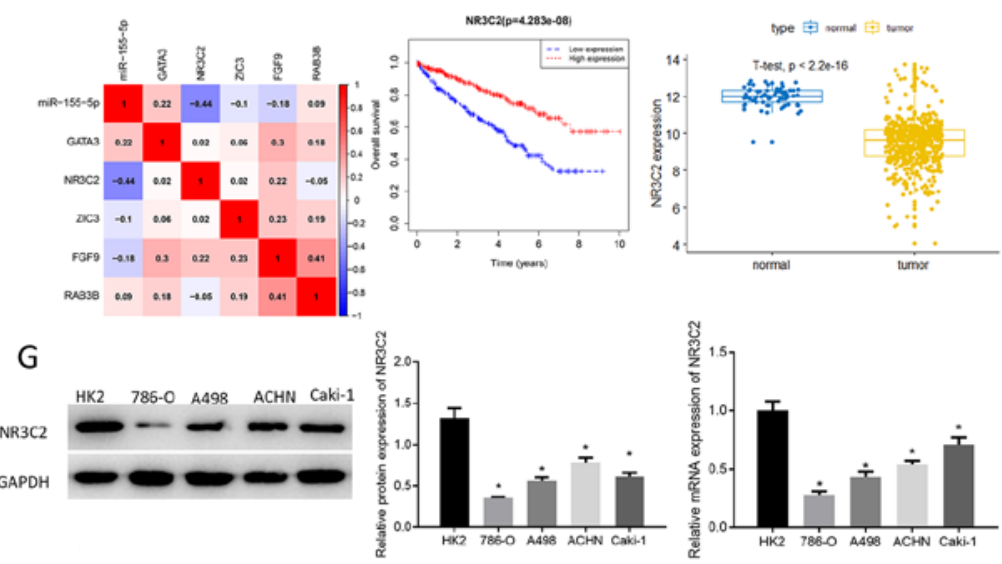

$\mathrm{H}$

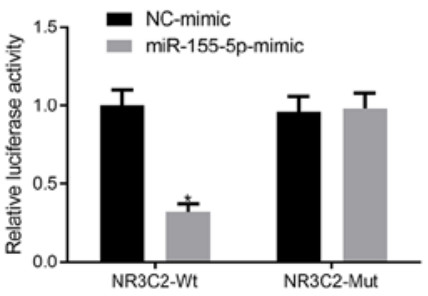

I
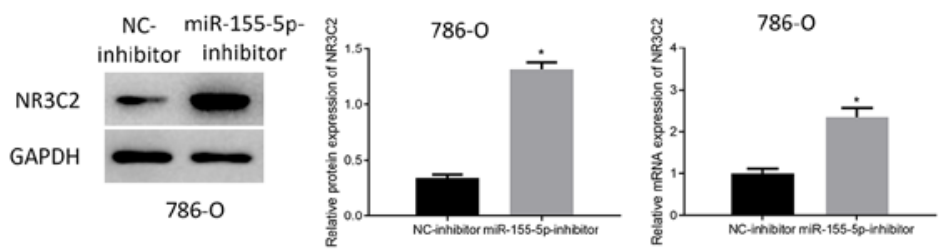

J

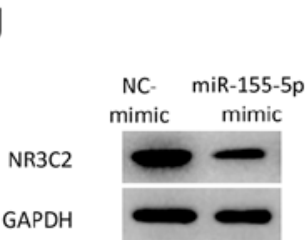

Caki-1
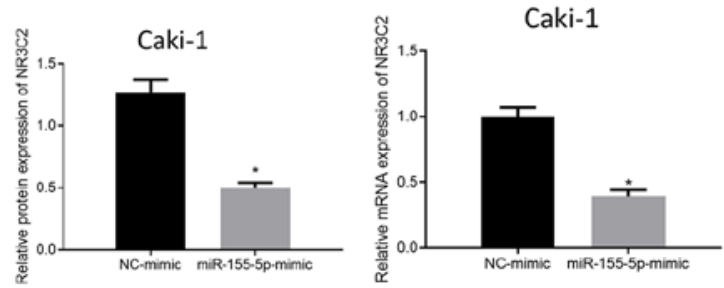

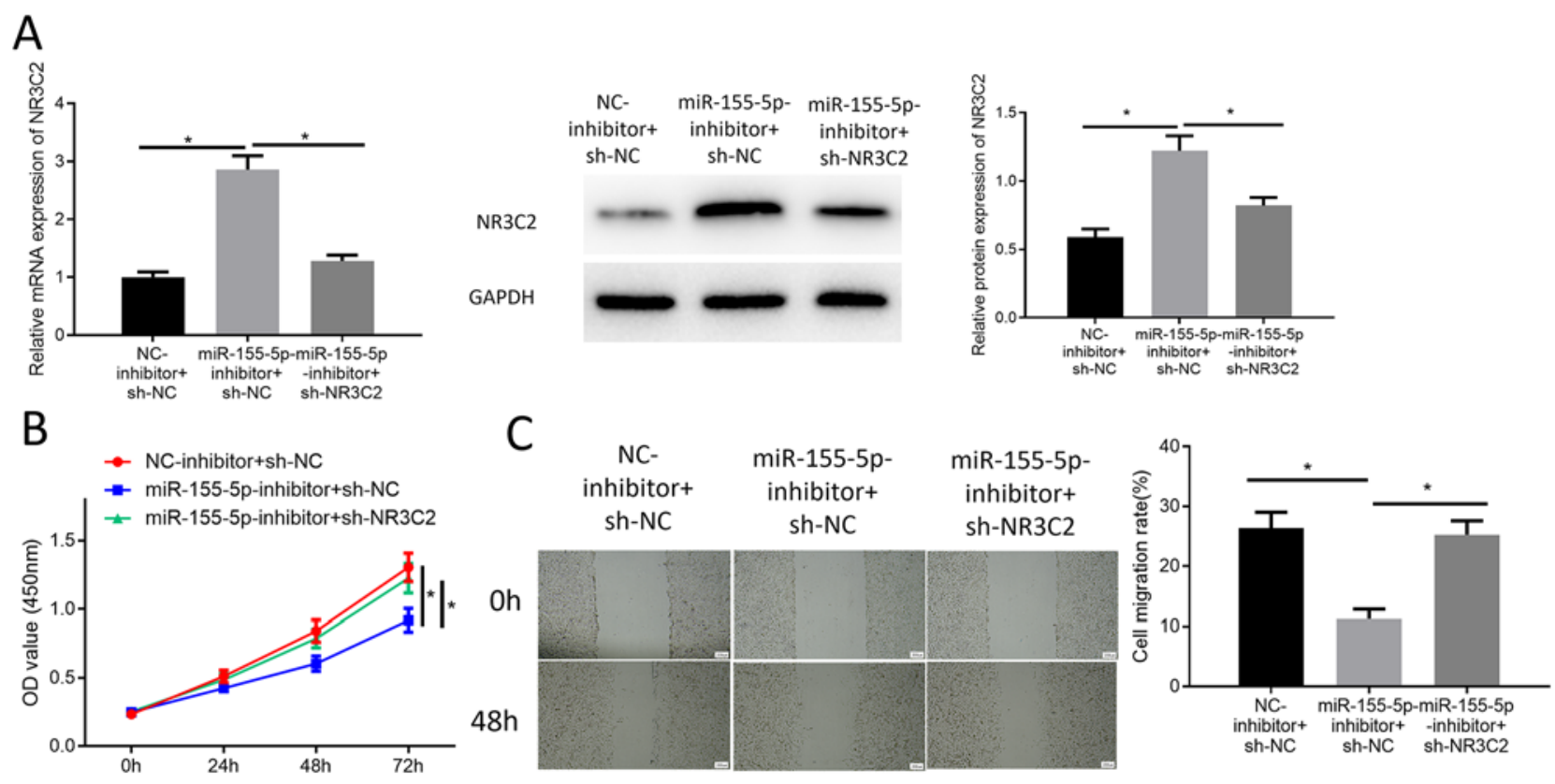

D NCinhibitor+ sh-NC

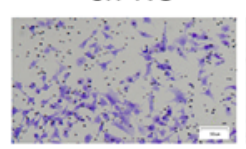

miR-155-5pinhibitor+ sh-NR3C2

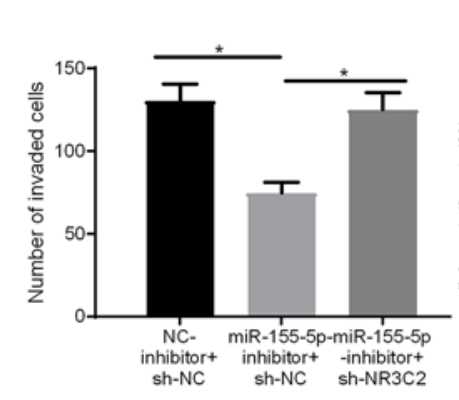

Fig. 4. Silenced microRNA-155-5p inhibits the malignant progression of ccRCC by targeting NR3C2. A mRNA and protein expression levels of NR3C2 in 786-O cells. B Proliferative status in the 3 groups. C Migratory status in the 3 groups $(\times 40)$. D Invasive status in the 3 groups $(\times 100)$. E Apoptosis degree in the 3 groups. $*$ denotes $p<$ 0.05 . NC, negative control.

Knockdown of NR3C2 reversed the inhibitory effect of the microrNA-155-5p-inhibitor on ccRCC cell migration (Fig. 4C). Similarly, inhibition of microRNA-155-5p significantly reduced the number of invaded cells, while knockdown of NR3C2 reversed the inhibitory effect of microRNA-155-5p-inhibitor on ccRCC cell invasion (Fig. 4D). In addition, inhibition of microRNA-155-5p remarkably increased the apoptosis rate, while NR3C2 knockdown overturned the promoting effect of microRNA 155-5p-inhibitor on ccRCC cell apoptosis (Fig. 4E). These findings indicated that microRNA-155-5p could suppress the malignant progression of ccRCC by targeting NR3C2.

\section{Discussion}

More and more studies confirmed that microRNAs are often dysregulated in ccRCC and play a part in promoting or inhibiting the progression of ccRCC. For instance, Chen et al. [15] proved that the expression level of microRNA-645 is notably upregulated in ccRCC tissue compared to adjacent tissue, and downregulation of microRNA-645 can constrain the malignant properties of ccRCC cells and promote cell apoptosis. Maolakuerban et al. [16] also uncovered that microRNA-200C-3p is under-expressed in ccRCC tissue, and microRNA-200C-3p can suppress the proliferation, invasion, and migration of 
ccRCC cells. In this study, it was confirmed that microRNA-155-5p was also upregulated in ccRCC, and it was further found through cell biological functional experiments that silenced microRNA-155-5p inhibited the proliferation, migration, and invasion of ccRCC cells while induced cell apoptosis. However, overexpression of microRNA-155-5p could boost the phenotypes of ccRCC cells while restraining cell apoptosis. These results strongly suggested that microRNA-155-5p is an oncogenic factor in ccRCC and can induce malignant progression of ccRCC cells.

$\mathrm{NR} 3 \mathrm{C} 2$ is defined as a gene for the mineralocorticoid receptor (MR), located on human chromosome 4q31.131.2 , and encodes the MR. The MR is expressed in a variety of tissues, including the colon, kidney, heart, sweat glands, etc. In epithelial tissue, MR activation results in sodium reabsorption by regulating the expression of ions and water-related transport proteins, thereby increasing extracellular volume to maintain a normal salt concentration in vivo [17]. However, recent studies found that NR3C2 is not only associated with memory loss [18], pseudohypoaldosteronism [19], and hypertension [20], it also plays a critical regulatory part in the development of various cancers through inhibition by a variety of microRNAs. For example, Zhao et al. [21] confirmed that microRNA-1204 can boost glioblastoma proliferation and reduce cell apoptosis by targeting NR3C2. Guo et al. [22] demonstrated that microRNA-454 plays an oncogenic role in oral squamous-cell carcinoma by targeting NR3C2 expression. However, in the present study, the downstream targets of microRNA-155-5p were mined by bioinformatics prediction, revealing that there is a binding relationship between NR3C2 and microRNA-155-5p, which presented a negative correlation in ccRCC tissues. Subsequently, it was confirmed that NR3C2 was indeed under-expressed in ccRCC cells. Then, the dual-luciferase assay also verified that microRNA-155-5p and NR3C2 could bind to each other. Further studies expressed that silenced microRNA-155-5p could lead to the upregulation of ccRCC mRNA and protein expression, while overexpression of microRNA-155-5p could lead to the opposite result. Finally, the rescue experiments indicated that downregulation of NR3C2 reversed the inhibitory effects of silenced microRNA-155-5p on proliferation, migration, and invasion of ccRCC cells, as well as the promoting effect on cell apoptosis. This suggested that microRNA-155-5p could promote the malignant progression of ccRCC cells by targeting NR3C2.

Collectively, in this study, we uncovered that microRNA-155-5p was an oncogenic factor of ccRCC and in- duced malignant progression of ccRCC cells. The tumorpromoting function of microRNA-155-5p in ccRCC was realized through inhibition of the expression of NR3C2. This study lays a certain foundation for microRNA$155-5 \mathrm{p}$ as a potential therapeutic target in ccRCC patients. However, there are still shortcomings in this study. For example, the differential expression of microRNA$155-5 p$ was not verified in clinical tissue and the regulatory function of microRNA-155-5p on the progression of ccRCC was not studied in vivo. These need to be further explored in subsequent studies.

\section{Statement of Ethics}

An ethics statement was not required for this study type as no human or animal subjects or materials were used.

\section{Conflict of Interest Statement}

The authors have no conflicts of interest to declare.

\section{Funding Sources}

This research received no specific grant from any funding agency in the public, commercial, or not-for-profit sectors.

\section{Author Contributions}

C.Q. Yan contributed to the conception of the study. P.F. Wang conducted the literature search. C.F. Zhao acquired the data. G.W. Yin wrote the initial draft of the paper. X. Meng analyzed the data. C.Q. Yan, L. Li, and S.Y. Cai discussed the results and revised the manuscript. B. Meng gave the final approval of the version to be submitted.

\section{Data Availability Statement}

Extra data can be accessed from the corresponding author on reasonable request. 


\section{References}

1 Ljungberg B, Hanbury DC, Kuczyk MA, Merseburger AS, Mulders PF, Patard JJ, et al. Renal cell carcinoma guideline. Eur Urol. 2007 Jun;51(6):1502-10.

2 Gupta K, Miller JD, Li JZ, Russell MW, Charbonneau C. Epidemiologic and socioeconomic burden of metastatic renal cell carcinoma (mRCC): a literature review. Cancer Treat Rev. 2008 May;34(3):193-205.

3 Li Y, Guan B, Liu J, Zhang Z, He S, Zhan Y, et al. MicroRNA-200b is downregulated and suppresses metastasis by targeting LAMA4 in renal cell carcinoma. EBioMedicine. 2019 Jun;44:439-51.

4 Wei R, Ye X, Zhao Y, Jia N, Liu T, Lian W, et al. MicroRNA-218 inhibits the cell proliferation and migration in clear cell renal cell carcinoma through targeting cancerous inhibitor of protein phosphatase 2A. Oncol Lett. 2019 Mar; 17(3):3211-8.

5 Leibovich BC, Lohse CM, Crispen PL, Boorjian SA, Thompson RH, Blute ML, et al. Histological subtype is an independent predictor of outcome for patients with renal cell carcinoma. J Urol. 2010 Apr;183(4):1309-15.

6 Ljungberg B, Bensalah K, Canfield S, Dabestani S, Hofmann F, Hora M, et al. EAU guidelines on renal cell carcinoma: 2014 update. Eur Urol. 2015 May;67(5):913-24.

7 He L, Hannon GJ. MicroRNAs: small RNAs with a big role in gene regulation. Nat Rev Genet. 2004 Jul;5(7):522-31.

8 Krol J, Loedige I, Filipowicz W. The widespread regulation of microRNA biogenesis, function and decay. Nat Rev Genet. 2010 Sep; 11(9):597-610.
9 Xie Y, Chen L, Gao Y, Ma X, He W, Zhang Y, et al. miR-363 suppresses the proliferation, migration and invasion of clear cell renal cell carcinoma by downregulating S1PR1. Cancer Cell Int. 2020;20:227.

10 Liu F, Chen Y, Chen B, Liu C, Xing J. MiR-935 promotes clear cell renal cell carcinoma migration and invasion by targeting IREB2. Cancer Manag Res. 2019;11:10891-900.

11 Chen G, Wang D, Zhao X, Cao J, Zhao Y, Wang F, et al. miR-155-5p modulates malignant behaviors of hepatocellular carcinoma by directly targeting CTHRC1 and indirectly regulating GSK-3beta-involved Wnt/betacatenin signaling. Cancer Cell Int. 2017;17: 118.

12 Li N, Cui T, Guo W, Wang D, Mao L. MiR155-5p accelerates the metastasis of cervical cancer cell via targeting TP53INP1. Onco Targets Ther. 2019;12:3181-96.

13 Shi SS, Zhang HP, Yang CQ, Li LN, Shen Y, Zhang YQ. Exosomal miR-155-5p promotes proliferation and migration of gastric cancer cells by inhibiting TP53INP1 expression. Pathol Res Pract. 2020 Jun;216(6):152986.

14 Wu M, Duan Q, Liu X, Zhang P, Fu Y, Zhang $Z$, et al. MiR-155-5p promotes oral cancer progression by targeting chromatin remodeling gene ARID2. Biomed Pharmacother. 2020 Feb;122:109696.

15 Chen J, Shu Y, Yu Q, Shen W. MicroRNA-645 promotes cell metastasis and proliferation of renal clear cell carcinoma by targeting GK5. Eur Rev Med Pharmacol Sci. 2017 Oct;21(20): 4557-65.
16 Maolakuerban N, Azhati B, Tusong H, Abula A, Yasheng A, Xireyazidan A. MiR-200c-3p inhibits cell migration and invasion of clear cell renal cell carcinoma via regulating SLC6A1. Cancer Biol Ther. 2018 Apr 3;19(4): 282-91.

17 Fan YS, Eddy RL, Byers MG, Haley LL, Henry WM, Nowak NJ, et al. The human mineralocorticoid receptor gene (MLR) is located on chromosome 4 at q31.2. Cytogenet Cell Genet. $1989 ; 52(1-2): 83-4$.

18 Chelghoum M, Guenane H, Harrat M, Yousfi M. Total tocopherols, carotenoids, and fatty acids content variation of Pistacia atlantica from different organs' crude oils and their antioxidant activity during development stages. Chem Biodivers. 2020 Sep;17(9):e2000117.

19 Barone Pritchard A, Ritter A, Kearney HM, Izumi $\mathrm{K}$. Interstitial $4 \mathrm{q}$ deletion syndrome including NR3C2 causing pseudohypoaldosteronism. Mol Syndromol. 2020 Jan;10(6):32731.

20 Cui Z, Xu J, Jiang W. NR3C2 gene polymorphism is associated with risk of gestational hypertension in Han Chinese women. Medicine. 2019 Dec;98(50):e18215.

21 Zhao X, Shen F, Ma J, Zhao S, Meng L, Wang $\mathrm{X}$, et al. CREB1-induced miR-1204 promoted malignant phenotype of glioblastoma through targeting NR3C2. Cancer Cell Int. 2020;20: 111.

22 Guo JY, Wang YK, Lv B, Jin H. miR-454 performs tumor-promoting effects in oral squamous cell carcinoma via reducing NR3C2. J Oral Pathol Med. 2020 Apr;49(4):286-93. 